XVII.

Aus der II. medizinischen Klinik München (Direktor: Professor Friedrich Müller) und der Prosektur des Krankenhauses R. d./J.

\title{
Über Blutbildung in Milz und Leber bei experimentellen Anämien.
}

$$
\text { Von }
$$

Dr. A. von Domarus.

(Mit Tafel II, III).

Seit den Arbeiten Neumanns über die Bildungsstätte der roten Blutkörperchen gilt allgemein das Knochenmark als das allein blutbildende Organ des erwachsenen Körpers, wenigstens für die Zeiten normalen Blutverbrauchs und normaler Blutneubildung. Es fragt sich aber, und diese Frage ist bereits mehrfach aufgeworfen worden, ob unter pathologischen Verhältnissen, d. h. bei gesteigertem Blutzerfall oder -Verlust und dementsprechend gesteigerter Neubildung das Knochenmark allein diese Funktion erfüllt, oder ob unter bestimmten Verhältnissen auch noch andere Organe unterstïtzend oder vikariierend an der Blutregeneration mitwirken können.

Anhaltspunkte hierfür sind schon seit langer Zeit bekannt. So weiß man, daß nach stark anämisierenden Einflüssen die Milz Veränderungen erfahren kann, die auf blutbildende Tätigkeit hinweisen.

Zuerst zeigten Bizzozero und Salvioli 1881 an der Hand ron Versuchen an Meerschweinchen, daß es gelingt, im Gefolge von Aderlässen bei Tieren eine Unwandlung der Milzpulpa zu erzielen, die für eine lienale Hämatopoese spricht. Über die näheren histologischen Details lassen sich die beiden Forscher nicht weiter aus. $\mathrm{Zu}$ ähnlichen Resultaten gelangten Howell (1888) mit Hilfe von Aderlaßanämien bei Katzen, P op off (1892) mit Blutgiftanämien; auf demselben Wege My a , Fo à vermittelst Unterbindung der Vena splenica ${ }^{1}$ ). Elia sberg ${ }^{2}$ )

1) Grawitz, Pathologie des Blutes, 1906. Nägelì, Blutkrankheiten und Blutdiagnostik, Leipzig 1907. A. Noll, i. Ergebn. d. Physiol., 2. Jahrg., 1903. A. Oppel, Zentralbl. f. allgem. Pathol, 1892.

2) Eliasberg Miron, Blutbildung in d. Milz, I.-D., Dorpat 1893. Archiv f. experiment. Pathol. a. Pharmakol. Bd. 58. 
beobachtete unter Barfurths Leitung an Hunden und Katzen die interessante Tatsache, daß die als Hămatopoese anzusehenden Veränderungen der Milz im Anschluß an Aderlässe in besonders hohem Maß sich nach Resektion eines Stückes der Milz fanden; und zwar sah Eliasberg massenhaft kernhaltige rote Blutkörperchen in den Pulpasträngen, besonders in der Nachbarschaft der Venen. Grü nberg und Dominiei kamen zu ähnlichen Resultaten. Auch $H$ einz ${ }^{1}$ ) beobachtete an Kaninchen, die unter der Wirkung von Blutgiften standen, nnter bestimmten Verhältnissen eine zweifellose Beteiligung der Milz an der Erythropoëse.

Dabei kommt er zu dem merkwïrdigen Resultat, daß man hauptsächlich dann positive Ergebnisse erhält, wenn der Untergang der roten Blutkörperchen ein sehr rapider ist. Dies ist die einzige Angabe, über die Abhängigkeit des Zustandekommens derartiger extramedullärer Veränderungen vom „klinischen" Verlauf der Anämie.

Beim Menschen wurden lienalen Erythropoese von Foà und Pellacani bei sekundären Anämien, von $A$. Wolff, Engel, Erich Meyer und A. Heinek e bei perniciöser Anämie; ferner u. a. von Kurjuweit bei Knochencarcinom, von $\mathrm{Nau}$ werck bei Osteosklerose konstatiert.

Über die Rolle der menschlichen Leber bei der Blutbildung im extrauterinen Leben war bisher wenig bekannt und die spärlichen Angaben beziehen sich auf myeloide Leukämien, nicht auf Anämie. Erst in der letzten Zeit wurden einzelne Beobachtungen publiziert, wonach sowohl erythro- wie leukoblastische Funktionen der Leber bei verschiedenen, das Blut schädigenden Erkrankungen zuzukommen seheinen.

Hierher gehören 2 Fälle Askanazys von Carcinommetastasen im Knochenmark und ein. Fall von allgemeiner Osteosclerose. Die dabei als weißliche Herde imponierenden Veränderungen im Lebergewebe waren Zellanhäufungen in den sackartig erweiterten Leberkapillaren und bestanden aus typischen Knochenmarkselementen. Bezüglich der Deutung dieser Veränderungen wurde in der Mehrzahl der Fälle eine einfache Einschwemmung von Knochenmarksgewebe supponiert, ohne daran weitere Erörterungen zu knüpfen.

In jüngster Zeit haben nun Erich Meyer und Albert Heineke ${ }^{2}$ ) an der Hand einer gröBeren Zabl von klinisch beobachteten und zur Autopsie gelangten Fällen von schwerer Anämie auf die dabei häufig zu konstatierenden Veränderungen in Milz und Leber

1) R. Heinz, Lehre von der Funktion der Milz, Virch. Arch. 1902, 168. Cf. auch die Arbeiten von $\mathrm{M}_{\text {alassez }}$ und $\mathrm{P}$ icard (Comptes rendus 1875, T. 81) L a udenbach (Zentralbl. f. Physiol. 1895, Bd. 9) und Freyer, I.-D., Königsberg 1872 .

2) Verhandlungen der Deutsch. pathol. Gesellsch., Meran 1905; Dentsches Arch. f. klin. Mediz., Bd. 88 . 
hingewiesen. Die bei manchen Anämien vorhandene auffallende Ähnlichkeit von Leber und Milz mit embryonalen Organen fassen sie als einen Beweis für die compensatorischen Bestrebungen des Organismus gegenüber primärer Blutsehädigung auf und sehen in diesem Rückschlag ins Embryonale eine nützliche Abwehrungsregel des Körpers. Aus dieser einheitlichen Basis herans erklärt sich ihnen der bei manchen primären Anämien zu erhebende "leukämoide" Blutbefund, indem sich bei diesen Fällen die vikariierenden, das Knochenmark ersetzenden Organe nicht nur auf die Erythropoese beschränken, sondern auch leukoblastisehe Funktionen übernehmen.

Die Annahme E. Meyers und Heinekes, daß die genannten Organveränderungen als kompensatorisch regenerative aufzufassen sind, stuttzt sieh auf die Analogie mit dem Verhalten derselben Organe beim normalen Embryo, bei dem in Leber und Milz zu bestimmten Zeiten lebhafte Blutzellenbildung stattfindet. Die Autoren nehmen an, daß bei perniciöser Anämie eine toxische Substanz das Blut schädigt und zu gesteigertem Blutzerfall führt, und daß bei genügender Regenerationsikraft des Organismus die Organe, die beim Embryo blutbildend sind (Milz, Leber), auf den gesteigerten Bedarf an neuem Blut mit dem Knochenmark zusammen reparatorisch reagieren. Diese Annahme war einer experimentellen Prüfung zugänglich und es mubte untersucht werden, ob beim Tier, das künstlich anämisch gemacht worden war, ein ähnliches Verhalten derblutbildenden Organe sich hervorbringen lieb. Falls diese Frage in bejahendem Sinne beantwortet werden konnte, drängte sich sofort die weitere Frage auf, ob aus dem klinischen Verlauf der Anämie sich die Bedingungen erklären lieben, unter denen eine derart gesteigerte Blutbildung in sämtlichen blutbildenden $\mathrm{Or}$ ganen zustande kommt.

Diese Aufgabe soll die folgende Untersuchung erfüllen, nachdem orientierende Versuche von Morris 1 ) bisher nicht zu ganz eindeutigen Resultaten geführt hatten.

Als Versuchstiere wurden Kaninchen benutzt, zumal bereits Bizzozero and Salvioli darauf hinweisen, daß die Kaninchen insofern gegenüber den anderen kleinen Säugetieren eine Ausnahmestellung einnehmen, als sie bereits kurz nach der Geburt normalerweise keinerlei Blutbildung in der Milz mehr zeigen. Wenn aber

1) Inzwischen sind die Untersuchungen von Morris wieder aufgenommen und in The Johns Hopkins Hospital Bulletin. Vol. XVIII, June-Jule 1907 publiziert. 
die genannten Forscher für die Zwecke der experimentell zu erzeugenden Blutbildung in der Milz die Kaninchen als ungeeignete Versuchstiere bezeichnen, so kann dem auf Grund der folgenden Untersuchungen nicht beigepflichtet werden.

Bekanntlich bieten sich, um Tiere künstlich anämisch zu machen, zwei Wege, der Aderlaß und die hämolytischen Gifte. Es liegt auf der Hand, daß mit ersterem, falls man auf Organveränderungen als Folgen der Anämie fahndet, zweifellos klarere Verhältnisse gesehaffen werden, doch ist andererseits zu bedenken, daß man durch Aderlässe, die sich ja am Tiere nur in beschränkter Zabl anwenden lassen, keineswegs das Äquivalent der langen, über Wochen und Monate ausgedehnten mensehlichen perniciösen Anämien erwarten darf. Auch ist daran zu erinnern, daß gerade für die sehweren und typisch perniciösen Anämien des Menschen ätiologisch toxisch wirkende Faktoren zu supponieren sind, wie sie beispielsweise das Bothriocephalusgift darstellt.

Übrigens wurde der Versuch gemacht, dureh Ansetzen von Blutegeln den Tieren einfache Aderlaßanämien beizubringen. Doch sind meine bisherigen Versuche mit dieser Methode durchaus unbefriedigend ausgefallen. Zunächst war es überaus schwierig, die Blutegel zum Saugen zu veranlassen nnd es bedurfte einer großen Zahl von Versuchen, um schließlich mit vereinzelten Exemplaren zum Ziele zu gelangen. Andererseits wurde an den drei Tieren, denen auf diese Weise Blut entzogen wurde, die merkwürdige Beobachtung gemacht, daß sie, obschon das verlorene Blutquantum unmöglich hierfür ausreichend sein konnte, innerhalb der nächsten Stunden eingingen. Da sich hierfür keine anatomisch nachweisbare Erklärung finden ließ, dürfte za prüfen sein, ob es sich .etwa um akut toxische Wirkungen handelt, die von den Blutegeln ausgehen. Jedenfalls ist diese Methode protrahierte Anämien zu erzielen wenigstens für Kaninchen nicht sehr aussichtsreich. Auch durch Aderlässe erhielten wir keine langdauernden Anämien.

Es wurden daher ausschließlich Blutgifte verwendet und zwar haupt-. sächlich das Phenylhydrazinacetat 1), daneben auch das Pyrogallol, und das Pyrodin (Acetyl-phenylhydrazin), in vereinzelten Fällen das Hydroxylamin.

Die Einverleibung der Giftlösung erfolgte fast ausnahmslos subkutan unter die Rückenhaut. Die Lösung des Phenylhydrazinacetates in 1 proz., die des Pyrogallols in 10 proz. Lösung. Hautabszesse wurden dabei nur ausnahmsweise beobachtet, wogegen derbe Infiltrate der Haut, besonders bei Tieren mit langdauernden Anämien sich nicht vermeiden ließen.

In vereinzelten Fällen wurde die Giftlösung per os gegeben. Doch wurde von dieser Methode umso lieber Abstand genommen, als, abge-

1) S. auch Kaminer u. Rohnstein, Berl. klin. Wochenschr. 1900, Nr. 31. 
sehen von der Verschiedenheit der anzuwendenden Dosen die erwarteten Veränderungen keine Abweichungen von denjenigen der subkutan vergifteten Tiere aufwiesen - (dies wird auch von Tallquist bestätigt) - andererseits aber die tägliche Anwendung der Schlundsonde das Leben des Tieres jedesmal in Gefahr bringt. Die Einverleibung des Giftes in Pillenform wurde nicht versucht.

Betreffs der jedesmal anzuwendenden Dosis war allein der täglich erhobene Blutbefund maßgebend, besonders für die chronisch verlaufenden Fälle. Diese . Vorsicht ist nicht nur mit Rücksicht auf die individuell verschiedene Giftempfindlichkeit äußerlich scheinbar gleicher Tiere notwendig, sondern wegen der auch von Tallquist ${ }^{1}$ ) hervorgehobenen, rasch zunehmenden Gewöhnung der Tiere an ein Blutgift erforderlich. So konnte konstatiert werden, daß eine Giftdosis, die genïgte, um Hämoglobin und Zahl der Erythrocyten auf einen bestimmten Wert herab. zudrücken, vergrößert werden mußte, um den Hämoglobinwert nicht wieder sofort ansteigen zu lassen.

Zuvor noch einige kurze Bemerkungen über das normale Kaninchenblut, so weit es für die folgenden Untersuchungen erforderlich ist.

Die Zählung der roten Blutkörperchen ergab beim gesunden erwachsenen Tier Werte zwischen 4,470000 und 8,40000; die Zahl der pseudoeosinophilen Leukocyten war auffallend schwankend, so daB man die physiologische Breite, innerhalb deren sich ihre Werte bewegen, zwischen 3800 und 13100 legen muß. Die Werte für das.Hämoglobịn sind wohl bäufig nicht mehr völlig normal, indem eine mehr oder weniger ausgeprägte "Stallanämie" den Prozentgehalt bis auf 70 herabsinken läßt, während die übrigen anscheinend der Norm entsprechenden Werte zwischen 100 und 120 liegen. Es wurde ein Sahlisches Hämoglobinometer benutzt, das ab und zu mit einem kontrollierten A pparat korrigiert wurde.

Ein Punkt erscheint bezuiglich der Technik der Blutuntersuchung erwähnenswert. Unter dem Einfluß verschiedener Blutgifte, in hervorragendem Maße aber bei Vergiftungen mit Phenylhydrazin und seinen Derivaten zeigen die roten Blatkörperchen außer Schrumpfung eine eigentümliche Köruchenbildung, auf die zuerst von $\mathrm{Hein} \mathrm{z}^{2}$ ) aufmerksam gemacht worden ist. Dieselbe ist nur im frischen resp. nicht fixierten Präparate sichtbar und z. B. mit Methylenblau oder Methylviolett leicht darstellbar, bei dieser Färbung sieht man oft in der Mitte eines Erythrocyten eventuell auch an seiner Peripherie haftend ein, seltener mehrere „Blaukörner". Im ersteren Falle, wo ein derartiges Blaukorn im Innern eines roten Blutkörperchens sitzt, könnte es bei flüchtiger Betrachtung für einen Kern gehalten werden, von dem es sich aber a. a. durch Fehlen jeder Struktur unterscheidet. Ihr Auftreten ist nun

1) Tallquist, Experimentelle Blutgiftanămien, Berlin 1900.

2) H einz, Experiment. Patholog. u. Pharmakolog., ferner Zieglers Beitr. t901, Bd. 29 und Virch. Arch., Bd. 122. 
insofern für die Zählung der Leukocyten von Bedeutung, als sie, wenn sie sehr zahlreich sind, in einer Zei B-Thomaschen Zählkammer event. von weißen Blutzellen schwer zu unterscheiden sind, zumal sie bei Tinktion der Zellkerne mit der ïblichen Essigsäure-Gentiana-ViolettLösung zum Farbstoff in hohem Grade Verwandschaft zeigen. Es bedarf daher gerade die Leukocytenzählung in vorgeriückten Stadien der Anämie sehr großer Aufmerksamkeit, sollen nicht erhebliche und für die Beurteilung der folgenden Befunde schwerwiegende Irrtiimer unterlaufen.

Mit Riicksicht auf die Morphologie des Kaninchenblutes soll noch hervorgehoben werden, daß in keinem Fall unter normalen Verhältnissen Myelocyten ${ }^{1}$ ) im kreisenden Blut gesehen wurden. Es wurden von sämtlichen 23 Fällen im normalen Stadium Abstrichpräparate auf ihre verschiedenen Zellarten hin untersucht. Dagegen finden sich, und darauf ist auch von anderer Seite hingewiesen worden, bereits unter normalen Verhältnissen regelmäßig einzelne polychromatophile Erythrocyten in einem oder dem anderen Gesichtsfeld.

Hier sei die prozentuale Zusammensetzung der weißen Blutzellen aus Abstrichpräparaten 3 normaler ausgewachsener Tiere eingeschaltet.

Pseudoeos. polynucl. Leuk. 47,1 resp. 64,1 und 49,4 Proz.

Ungranulierte (Lymphocyten) 39,5 resp. 25,1 und 44,1 Proz.

Mastzellen 10,0 resp. 9,0 und 6,4 Proz.,

Eosin. polynuel. Leuk. 3,1 resp. 1,0 und 0 Proz.

Demnach überwiegen auch bei Kaninchen im ausgewachsenen $\mathrm{Zn}-$ stand die Granulocyten iuber die Lymphocyten.

Im folgenden sollen aus der Gesamtzahl der vorliegendèn Versuchsprotokolle nur einzelne, typische Fälle herausgegriffen werden. Im ganzen wurden 23 Versuche angestellt.

\section{Akute und subakute Anämien.}

1) Weibl. Kaninchen Nr. 2. Gewicht 1610 g. Phenylhydrazin in 1 Proz. Lösung, subkutan.

Blutbefund:

3. III. 06. 6500000 R.; 110 Proz. Hb (Sahli), 6000 L. 0,1 4. III. 06. 1। Uhr a.m. $4000000 \mathrm{R} ; 70 \mathrm{Proz} . \mathrm{Hb} \quad 19500 \mathrm{~L}$.

Morph : zahlr. polychromat. Erythrocyten, ebenso viel punktierte E.

Im frischen Präparat viel "Blaukörner".

4. III. 8 Uhr. 4000000 R.; 55 Proz. Hb 11200 L. Morph.: dasselbe Bild, nur sind polychrom. und punktiert E. noch stärker vermehrt.

5. III. 8 Uhr p. m. 5000000 R.; 65 Proz. Hb 7800 L. Morph.: keine kernhaltigen E. 0,02 Phen.

1) Zum Begriff Myelocyt wurden bei dieser Untersuchung natürlich auch buchtkernige Metamyelocyten (Y a p penheim) gerechnet. 
6. III. 7 Uhr p. m. 4120000 R.; 40 Proz. Hb; 16400 L. Morph. dasselbe Bild, vereinzelte kernhaltige E.: hochgradige Poikilo- und Anisocytose. Massenh. polychrom. E.

7. III. 12 Uhr m. 2460000 R.; 30 Proz. Hb; 11700 L.

7. III. 7 Uhr $m$. Durch Nackenschlag getötet.

Sektionsresultat: Blut in allen Organen chokoladenfarben. Leber dunkelbraunrot, brïchig; Milz etwa um ein Viertel größer als normal, die Kapsel durchscheinend, die Schnittfläche schwarzbraun, die Pulpa überquellend, Nieren erscheinen hyperaemisch, Marksubstanz hell, Rinde auffallend dunkel mit Stich ins bräunliche, gegen das Mark scharf abgesetzt. Herzmuskel blaß, brïchig.

Die mikroskopische Untersuchung ergibt trübe Schwellung und teilweise Verfettung der Leberzellen, reichliche Pigmentablagerung, besonders in den Endothelzellen, dagegen lassen Lebercapillaren, wie periportales Bindegewebe jegliche Art von Zellen vermissen, die auf Blutbildung, resp. Ansiedelung von Myeloidgewebe schließen lassen.

Im Abstrichpräparat ortho- und polychromatische Erythrocyten, kein e kernhaltigen roten Blutkörperchen, vereinzelte pseudoeosinophile Leukocyten, einzelne Lymphocyten.

Das gleiche gilt von der Milz. Reichliche Mengen von hauptsächlich intrazellulär gelegenem Pigment, starke Hyperaemie des ganzen Organes. Zahlreiche, mit zum Teil noch deutlich erkennbaren Erythrocyten vollgestopfte Makrophagen. Die Follikel sind unverändert nach Form und Größe. Keine kernhaltigen Erythrocyten, keine Myelocyten.

Im Abstrich massenhaft Makro- und Mikrolymphocyten.

Das Knochenmark erscheint diffus braunrot und hat auf der schnittfläche noch fettige Beschaffenheit.

Mikroskopisch erweist es sich als deutliches .Zellmark"; die normale wabige Struktur ist geschwunden, statt dessen finden sich gleichmäßig im ganzen Gesichtsfeld hauptsächlich Zellen von lymphoidem Habitus, auch psendoeosinophile und eosinophile Myelocyten. Die Zahl der Knochenmarksriesenzellen erscheint dagegen, verglichen mit einem normalen Knochenmarkspräparat, $\mathrm{z}$ we ifellos vermindert. Vereinzelt finden sich feinere und gröbere freie Pigmentscbollen.

Epikrise: Vergiftung mit Phenylhydrazin; getötet 5 Tage nach Beginn derselben. Keine Veränderungen in Milz und Leber.

Demnach bietet dieser Fall in bezug auf Regenerationsveränderungen in Milz und Leber ein negatives Ergebnis.

2) Weibliches Kaninchen Nr. 3. Gewicht 2000 g. Phen. 1 proz. subkutan.

Blutbefund:

9. III. 06. 6040000 R.; 105 Proz. Hb 10000 L. 7 Uhr p. m. 0,02 Phen. subkutan. 
10. III. 10 Uhr a. m. $5600000 \mathrm{R}$; 80 Proz. Hb 12000 L. Morph.: keine Normoblasten, dagegen polychrom. und gekörnte $\mathrm{E}$.

11. III. 0,02 Phen. subkutan.

12. III. 7 Uhr p. m. 4860000 R.; 75 Proz. Hb; 19800 L. Morph.: vereinzelte Normoblasten.

13. III. 5 Uhr p. m. 0,1 Phen.

14. III. 11 Uhr a. m. 3070000 R.; 55 Proz. Hb; 25300 L. 0,1 Phen.

15. III. 5 Uhr p. m. 3200000 R.; 40 Proz. Hb; 14200 L. 0,1 Ph. Morph. massenh. polychrom. und gekörnte E., sehr starke Anisound Poikilocytose. $A B$ und zu 1 Normoblast.

16. III. 12 Uhr m. 3160000 R.; 40 Proz. Hb; 10400 L. 0,1 Ph. subk. Morph.: Dasselbe Bild wie am Tage zuvor.

17. III. 12 Uhr m. 3020000 R.; 35 Proz. Hb; 17200 L. 0,1 Ph. subkutan.

Das Tier stirbt in der Nacht vom 17. III. zum 18. III. Die Sektion wird am 18. III. $10 \mathrm{Uhr}$ vorm. vorgenommen und hat folgendes Ergebnis:

Leber dunkelbraunrot, brïchig. Milz ziemlich stark vergrößert, blauschwarz auf Schnitt und Oberfläche, Pulpa hervorquellend braunschwarz. Herzmuskel brüchig, stark bräunlich, tingiert, in den Herzhöhlen einzelne chokoladenfarbene Coagula. Herzbeutelhöhle und Pleurahöhle enthalten wenig gelbliche seröse Flüssigkeit. Knochenmark dunkelbraunrot, läßt sich leicht aus der Markhöhle des Femur herausheben.

Mikroskopisch erweisen sich sämtliche genannten Organe von Pigment überschwemmt, besonders die Milz, die Leber etc. Bezüglich der erwarteten Veränderungen in Leber und Milz läßt auch dieser Fall, sowohl in Schnitt wie in Abstrichpräparaten völlig im Stich.

3) Männliches Kaninchen Nr. 15. Gewicht 2700 g, salzsaures Hydroxylamin subkutan.

Blutbefund: $0,025 \mathrm{sub}$.

21. VIII. 06. 12 Uhr m. 115 Proz.; 6680000 R.; 15000 L.

22. VIII. 12 Uhr m. 62 Proz. Hb; 4240000 R.; 25000 L. Morph.: Zahlreiche Mykrocyten, polyehrom. und getüpfelte E., vereinzelte kernhaltige E. 0,02 subk.

22. VIII. $4^{1 / 2}$ Uhr p. m. 55 Proz.

23. VIII. 11 Uhr a. m. 30 Proz. Hb; 25200000 R.; 28000 L. Morph.: Dieselben Veränderungen wie oben,. nur in weit höherem Grade Einzelne Myelocyten.

24. VIII.

25. VIII. wird das Tier tot im Stall aufgefunden.

Das Sektionsergebnis liefert den beiden vorigen Fällen völlig gleichende Befunde, auch mikroskopisch ist keine Abweichung zu konstatieren, nirgends finden sich als Blutbildungsherde anzusprechende Veränderungen. 
Ueber Blutbildung in Milz und Leber bei experimentellen Anămien. 327

Ganz so verhält es sich mit Kaninchen Nr. 14 (Gewicht 1900 g), das innerhalb von 4 Tagen (17. VIII. bis 20. VIII. 06) unter Phenylhydrazin-Wirkung akut zugrunde geht, wobei seine urspriinglichen Blutwerte $(5000000 \mathrm{R}$; 80 Proz. $\mathrm{Hb})$ auf $3200000 \mathrm{R}$ und 50 Proz. $\mathrm{Hb}$ herabsinken).

Auch hier ist das Sektionsresultat völlig negativ.

Diese Fälle beweisen, $d a \beta$ bei akutem Blutzerfall durch Einverleibung groBer Dosen eines hämolytischen Giftes eine schwere Anämie ohne Blutbildungsherde in Milz und Leber hervorgerufen wird.

Dem seien folgende Fälle gegenuiber gehalten.

\section{Chronische Vergiftungen.}

1) Männliches Kaninchen Nr. 20. Gewicht 2250 g. Phenylhydrazinacetat-Lösung $(0, \check{5}$ Proz.)

2. IV. 07. 7200000 R.; 100 Proz. Hb (Sahli); 6000 L. 0,01 subk.

3. IV. 5 Uhr p. m. 5120000 R.; 75 Proz. Hb. Morph.: Reichliche Blaukörner im frischen Präparat, einzelne punktierte E., keine Normoblasten 0,01 .

4. IV. 5 Ubr p. m. 4250000 R.; 60 Proz. Hb; 3700 L (?) 0,01 .

5. IV.

6. IV. 5 Uhr p. m. 3020000 R.; 60 Proz. Hb; 12000 L. Morph.: zahllose Blaukörner; im gefärbten Präparat viel punktierte und polychrom. Etythroc. Vereinzelte ortho- und polychrom. Normoblasten.

7. IV. 11 Uhr a. m. 0,015 .

8. IV. 4 Uhr p. m. 3300000 R.; 65 Proz. Hb; 14300 L. Morph.: ungefähr dasselbe Bild.

9. IV. 5 Uhr p. m. 3500000 R.; 65 Proz. Hb; 11200 L. Morph.: Ziemlich viel kernhaltige (keine Megaloblasten), fast in jedem Gesichtsfeld 1, ferner zahlreiche Makrolymphocyten. 0,01 subk.

11. IV. 07. 5 Uhr p. m. 4100000 R.; 60 Proz. Hb; $15250 \mathrm{~L}$. Morph.: Kernhaltige fast sämtlich verschwunden.

13. IV. 5 Uhr p. m. 3650000 R.; 55 Proz. Hb; 17800 L. 0,015 subk.

15. IV. 4 Uhr p. m. 4850000 R.; 75 Proz. Hb; - L. Morph.: in zwei Präparaten keine Normoblasten.

16. IV. 5 Uhr p. m. 4800000 R.; 70 Proz. Hb; 9600 L. Morph.: wie am 15. IV. 0,02 .

17. IV.

18. IV. 5 Uhr. 3500000 R.; 60 Proz. Hb; 21200 L. Morph.: wie am 16. IV.

19. IV. 5 Uhr. 0,02 subk.

20. IV. 5 Uhr p. m. 3200000 R.; 55 Proz. Hb; 16300 L. Morph.: sehr wenig Normoblasten, starke Veränderungen der Erythrocyten.

21. IV. $11 \mathrm{Uhr}$ a, m. Morph.: ziemlich viel Normoblasten, keine Megalobl.

22. IV. 5 Uhr p. m. 3180000 R.; 50 Proz. Hb; 14000 L.

24. IV. 5 Uhr p. m. 2800000 R. 46 Proz. Hb; 8700 L. Morph.: Normobl. verringert gegen 21. IV. 0,01 . 
26. IV. 5 Uhr p. m. 3100000 R.; 52 Proz. Hb; 9200 L. 0,015 subk.

27. IV.

28. IV. $10 \mathrm{Uhr}$ a. m. 0,03 subk.

29. IV. 4 Uhr p. m. 2520000 R.; 40 Proz. Hb; 10100 L. Morph.: ziemlich viel Normobl. 0,01 subk.

30. IV. 6 Uhr p. m. 2800000 R.; 45 Proz. Ḣb; 9700 L. 0,02 subk.

1. V. 6 Uhr p. m. 2600000 R.; 42 Proz. Hb; 9300 L. 0,03 subk.

2. V. 5 Uhr p. m. 2210000 R.; 35 Proz. Hb; 9900 L. Morph.: keine Normobl. 0,01 subk.

3. V. 4 Uhr p. m. 2850000 R.; 40 Proz. Hb; 4200 L. 0,02 .

4. V. 4 Uhr p. m. 2300000 R.; 37 Proz. Hb; 7200 L. Morph.: spärl. Normobl. 0,03 .

5. V. 10 Uhr a. m. 1950000 R.; 30 Proz. Hb; 14800 L. $0,01$.

6. V. 6 Uhr p. m. 2100000 R.; 30 Proz. Hb; 7700 L. Morph.: Normobl. fehlen in 2 Präparateu.

7. V. 5 Uhr p. m. 2100000 R ; 28 Proz. Hb; 9900 L. 0,01 .

8. V. 5 Uhr p. m. 2900000 R.; 25 Proz. Hb; 11200 L. Morph. spärlich hie und da 1 Normobl.

9. V. 11 Uhr a. m. 2900000 R.; 25 Proz. Hb; 4700 L. Morph. Normobl. gegen den 8. V. vermehrt.

10. V. 6 Uhr p. m. 2950000 R.; 30 Proz. Hb; 5400 L. 0,02 .

11. V. 6 Uhr p. m. 2120000 R.; 25 Proz. Hb; 9600 L. Morph. keine Normobl, keine Megalobl. 0,02.

12. $V$.

13. V. 5 Uhr p. m. 1850000 R.; 25 Proz. Hb; $7300 \mathrm{~L}$.

14. V. 6 Uhr p. m. 2100000 R.; 25 Proz. Hb; 8200 L. Morph. keine Normobl. 0,03 .

15. V. 6 Uhr p. m. 1520000 R.; 27 Proz. Hb; 9400 L. 0,02 .

16. V. 5 Uhr p. m. 1310000 R.; 25 Proz. Hb; 8900 L. 0,02. 7 Uhr p. m, wird das Tier tot aufgefunden.

Es stand also vom 2. IV. bis 16. V. unter der Wirkung des Blutgiftes. Die Auszählung der weißen Zellen im Blutpräparat hatte am 14. V. 6 Uhr p. m. ergeben:

$\begin{array}{lr}\text { Pseudeosin. polyn. } & 32,2 \text { Proz. } \\ \text { Lymphocyten } & 56,8= \\ \text { Mastzellen } & 9,1= \\ \text { Eosinophile L. } & -\overline{2}= \\ \text { Erythroblasten } & 2,2= \\ \text { Myelocyten } & -=\end{array}$

Zugleich mit diesem Tier wurde ein Kontrolltier einer möglichst gleichartig verlaufenden Anaemie unterworfen. Da das Ergebnis auch in anatomischer Beziehung analog ausfiel, so soll das Protokoll dieses 2. Tieres hier gleich angeschlossen werden.

Männliches Kaninchen Nr. 21. Gewicht 2550 g. Phenylhydrazinacetat subkutan.

Blutbefund:

2. IV. 07. 8400000 R.; 105 Proz. Hb (Sahli); 13000 L. 0,01 Phen.

3. IV. 07. 0,01 Phen. 
4. IV. 6 Uhr p. m. 6310000 R.; 85 Proz. Hb; 16000 L. 0,01.

5. IV. 4 Uhr p. m. $4500000 \mathrm{R}$.

6. IV. 4 Uhr p. m. 4120000 R.; 67 Proz. Hb; 15700 L. Morph: zahlreiche punktierte E., vereinz. Normobl.

7. IV. 12 Uhr m. 0,02 .

8. IV. 5 Uhr p. m. 3200000 R.; 60 Proz. Hb.

9. IV. 5 Uhr p. m. 3800000 R.; 60 Proz.; 12300 L. 0,01.

10. IV. 6 Uhr p. m. 3340000 R.; 60 Proz. Hb; 3400 L. (?) Morph.: viel Normobl., keine Megalobl.

11. IV. 5 Uhr p. m. 65 Proz. Hb;0,02.

12. IV. 6 Uhr p. m. 3410000 R.; 70 Proz. Hb; 21300 L. 0,02 .

13. IV. 5 Uhr p. m. 3000000 R.; 65 Proz. Hb; 10200 L. Morph.: Kein einzig. Normobl. in 2 Präp. $0,01$.

14. IV. 11 Uhr a. m. 3120000 R.; 0,02 .

15. IV. 5 Uhr p. m. 2890000 R.; 60 Proz. Hb; 8900 L. Morph.: Keine Normobl.

16. IV.

17. IV. 4 Uhr p. m. 3970000 R.; 70 Proz. Hb; 2200 L. Morph.: Einz. kernhaltig. rote B. 0,01 .

18. IV. 5 Uhr p. m. 4100000 R.; 70 Proz. Hb; 0,01

19. IV. 11 Uhr a.m. 4230000 R.; 65 Proz. Hb; 9700 L. $0,02$.

20. IV. 6 Uhr p.m. 3900000 R.; 60 Proz. Hb; 18900 L. Morph.? Keine Normobl.

21. IV.

22. IV. 5 Uhr p. m. 4620000 R.; 0,02 .

23. IV. 5 Uhr p. m. 3120000 R.; 54 Proz. Hb; 14700 L. Morph.: Hie und da 1 Normobl.

25. IV. 6 Uhr p. m. 3750000 R.; 63 Proz. Hb; 13900 L. $0,01$.

26. IV. 5 Uhr p. m. 3900000 R.; 65 Proz. Hb; 4900 L. $0,015$.

27. IV. 6 Uhr p. m. 60 Proz. Hb; $0,02$.

28. IV.

29. IV. 4 Uhr p. m. 3810000 R.; 60 Proz. Hb; 15200 L. $0,03$.

30. IV. 5 Uhr p. m. 2120000 R.; 50 Proz. Hb; 24600 L. Morph.: Zieml, viel Normobl.

1. $\mathrm{V}$.

2. V. 6 Uhr p. m. 2950000 R.; 50 Proz. Hb; 16300 L. 0,02 .

3. V. 6 Uhr p. m. 2140000 R.; 43 Proz. Hb; 11700 L. Morph.:

Keine Normobl. 0,02 .

4. V. 5 Uhr p. m. 2200000 R.; 37 Proz. Hb; 6700 L. Morph.: Keine Normobl. 0,01.

5. $\mathrm{V}$.

6. V. 6 Uhr p. m. 2470000 R.; 32 Proz. Hb; 9500 L. 0,02 .

7. V. 6 Uhr p. m. 2150000 R.; 30 Proz.; 4700 L. Morph.: Keine Normobl.

8. V. 5 Uhr p. m. 1950000 R.; 27 Proz. Hb; 8400 L. $0,015$.

9. V. 10 Uhr a. m. 30 Proz. Hb;0,01.

10. $\mathrm{V}$.

11. V. 6 Uhr p. m. 2100000 R.; 30 Proz. Hb; 11300 L. Morph.: Sehr zahlr. Normobl. 
12. V. 10 Uhr a. m. 1780000 R.; 25 Proz. Hb; 9700 L. $0,025$.

13. V. 5 Uhr p. m. 1230000 R.; 20 Proz. Hb; 6300 L. Morph.: Sehr spärl. Normobl.

Auszählung der weißen Zellen des Blutpräparates ergibt:

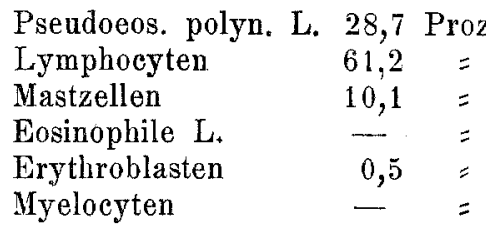

Das Tier wird getötet, nachdem es vom 2. IV. bis 13. V. die skizzierte Anaemie durchgemacht hatte.

Sektionsresultat der Tiere Nr. 20 und 21:

Sämtliche inneren Organe hochgradig anaemisch, Blut mißfarben, dünnflüssig, gerinnt langsạm. In Brust- und Bauchböhle wenig trïbe seröse Flüssigkeit. Herz sehr schlaff, brüchig, enthält braunrote Gerinnsel. Lungen o. B. Magenserosa am Fundus eigentümlich dunkel gefärbt. Die Blase enthält wenig mißfarbenen bräunlichen, trüben Urin, der starke Eiweißprobe gibt, und im Sediment ziemlich viel Nierenepithelien, rote Blutkörperchen und Zylinder enthält.

Die Milz ist stark vergrößert, besonders in ihrem Längsdurchmesser. Die Maße sind $8 \times 1 \times 0,4 \mathrm{~cm}$. Kapsel durchscheinend; Pulpa wenig: überquellend, fast schwarz.

Die Leber ist nicht wesentlich an Größe von der Norm abweichend, etwas brüchig, sonst in ihrer Konsistenz kaum verändert.

Auffallend an ihr ist - und darin liegt schon der makroskopische Hinweis auf vorhandene Veränderungen - die eigentümliche Zeichnung die derartige von ausgedehnten chronischen Anaemien herstammende Lebern an Oberfläche und Schnitt erkennen lassen.

Aus der menschlichen Pathologie erinnert am meisten die MuskatnuBleber an dies Bild, da Schnitt und Oberfläche wie gesprenkelt erscheinen und mit unzähligen dunkelroten teils circumscript punktförmigen, teils streifenförmigen Flecken übersät sind. Bemerkenswerter Weise läßt sich jedoch keine regelmäBige Verteilung dieser Partien nach Zentrum oder Peripherie der Acini beobachten.

Im mikroskopischen Bilde begegnet man einergeradezu frappanten Ähnlichkeit mit dem histologischen Habitus einer leukaemischen Leber und demjenigen fötaler Lebern in gewissen Stadien der Entwicklung (5-7 Monat).

Bei schwacher Vergrößerung erscheinen die Lebercapillaren derart mit Zellen angefüllt, daß an einer Stelle des Präparates, wo ein Acinus senkrecht zur Zentralvene geschnitten ist, von dieser ausgehend, die Zellzüge der intralobulären Venencapillaren wie dunkle Streifen erscheinen, die radiär vom Zentrum des Acinus nach der Peripherie ziehen.

Zugleich fällt in einem Übersichtsbild auf, daß die genannten Zellgebilde ungleich an Menge in jedem Acinus verteilt sind und stellenweise deutlich herdartige Anhäufuugen bilden. 
Nur ein Teil derselben liegt deutlich intracapillär derart, daß ein Zusammenhängen mit der Wand der Leberbalken resp. deren Endothel nicht nachweisbar erscheint.

Der größere Teil ist bei schwacher Vergrößerung von den Leberbalken selbst nicht abgrenzbar und scheint mit diesen in innigem Connex zu stehen. Das ist die Regel für die in Gruppen angeordneten Zellen. Sie liegen z. T. wie umschriebene Einzprengungen scheinbar innerhalb der Leberbalken besonders dort, wo mehrere benachbarte Leberbalken zusammenstoßen.

Endlich sieht man noch an einem Übersichtsbild, daß dort, wo deraríge Zellaggregate vorhanden sind, häufig das Lumen der von dem Leberbalken gebildete Capillare wie ausgebancht erscheint. Überhaupt sind die Lebercapillaren ïberall, wo dichtere Zellanhäufungen vorhanden sird, gegen die Norm erweitert.

Endlich ist noch zu konstatieren, daß unregelmäßig über jeden Schnitt verteilt sich Herde finden, in denen Leberzellen fast total verfettet sind, sodaß diese Stellen wie helle Liicken im übrigen Gewebe erscheinen, was umsomehr hervortritt, als dieselben scharf gegen die unveränderte Cmgebung abgegrenzt zu sein pflegen.

Die Bedeutung dieser verfetteter Partien dürfte untergeordneter Natur sein; vielleicht handelt es sich einfach um Residuen stark fetthaltigen Lebergewebes, wie es im Laufe dieser Untersuchungen wiederholt bei ganz jungen Tieren beobachtet wurde. wo event. die gesamte Leber eine derartige Beschaffenheit darbietet und eine wabige Struktur zu haben scheint.

Hinsichtlich des periportalen Gewebes waren die bisherigen Befunde inkonstant; stellenweise handelte es sich zweifellos um einen Zellreichtum, der über das Normale binausgeht, (so wurden vereinzelte Haufen von Normoblasten zusammen mit großen einkernigen Lymphoidzellen gefunden). An anderen Stellen konnte nichts derartiges konstatiert werden, werden, obschon gerade auf diese Veränderungen ein Hauptaugenmerk gerichtet wurde.

Betrachtet man einen solchen Leberschnitt mit der Immersionslinse, so ist man von der.Vielgestaltigkeit der verschiedenen Gesichtsfelder iuberrascht. Sowohl Anordnung wie Form der Zellen sind mannigfachen Variationen unterworfen. (Fig. 2).

Sehr deutlich erkennt man auch hier zunächst, daß ein Teil der besagten Zellen im Lamen der Lebercapillaren liegt, ohne in irgend einer engeren Verbindung mit Endothelien resp. Leberbalken zu stehen.

Der bei weitem größte Teil befindet sich dagegen nicht frei im Lumen der Capillare und dies dürfte gerade für die vorliegende Frage von besonderer Bedeutung sein.

An vielen Stellen erblickt man von dem Capillarlumen ausgebend, sinusartige Ausbuchtungen die wie Aussparungen der Leberbalken aussehen und iiberall, wo derartige Lakunen vorhanden sind, trifft man die genannten Zellanhäufungen an, so zwar, daß sehr zellreiche Haufen weiteren und tieferen Buchten zu entsprechen scheinen.

Was die Zellen selbst betrifft, so ist es schwer, sie mit wenigen Worten zu charakterisieren, da einmal verschiedene Zelltypen vorliegen, 
und anßerdem Zellen derselben Gruppe offenbar in verschiedenen Stadien ihrer Entwicklung fixiert wurden.

Hier soll bemerkt werden, daß, um ein einigermaßen sicheres Urteil über eine Zellart abgeben zu können, von der Methode der Abstrichpräparate in jedem Fall ein ausgiebiger Gebrauch gemacht wurde, was in vielen Beziehungen, speziell hinsichtlich der kernhaltigen roten Blutkörperchen von Bedeutung war.

Bezitglich der Methodik bei der Herstellung von Abstrichpräparaten sei kurz darauf hingewiesen, daß für eine möglichste Schonung der zellnlären Elemente beim Ausstreichen des Gewebsbreies sich die Anwendung zugeschnittener Kartonblättchen bewährt, deren Kante man mit dem Gewebssaft benetzt und darauf zart über einen Objektträger führt. Hierbei ist es wiederum zweckmäßig, das schief aufgesetzte Blatt, falls z. B. hauptsächlich an der Seite des spitzen Winkels das auszustreichende Material haftet in der Richtung des spitzen Winkels über den Objektträger zu verschieben; so wird jede Spur unnötiger Quetschung vermieden.

Die große Mehrzahl der in Frage kommenden Zellen hat ein ungranuliertes Protoplasma und gehört zum Lymphoidzellentypus. Ein ziemlich großer Prozentsatz unter ihnen sieht wie echte Lymphocyten aus - schmales, ziemlich stark basophiles, granulafreies Protoplasma, großer blasser Kern z. 'T. mit deutlichen Kernkörperchen.

An zweiter Stelle sind kernhaltige rote Blutkörperchen vertreten, deren typischer Radkern und ihr hämoglobinhaltiges Protoplasma die Erkennung sichert. Von größter Bedeutung sind dabei diejenigen Formen, die sich im Stadium der Kernteilung befinden; ihre Zahl ist allerdings nicht groß, doch finden sich in fast jedem Gesichtsfeld eine oder zwei Mitosen.

Hier muß bemerkt werden, daß aus der Auszäblung der verschiedenen Zellformen im Leberabstrichpräparat hervorgeht, daß die Zahl der Erythroblasten tatsächtich eine etwas größere ist, als eine Prüfung des Schnittpräparates vermuten läßit. Dies wird z. T. daraus erklärlich, dab ein Teil der kernhaltigen Erythrocyten mehrere Kerne besitzt, ein anderer Teil nicht mehr deutliche Radspeichenkerne erkennen läßt, sondern einen stark pyknotischen und daher strukturlosen Kern oder einen solchen mit Sprossungsfiguren usw. zeigt, während andererseits hochgradige Polychromasie des Protoplasmas die Abgrenzung gegen hämoglobinfreie Lymphoidzellen erschwert.

Als weitere Zellen sind solche zu nennen, die im Schnitt wie im Abstrich einen Buchtkern und breites granulafreies Protoplasma besitzen, jedenfalls gleichen sie den Ehrlich'schen großen mononucleären Leukocyten resp. den sogen. „Splenocyten (Türk-Pappenheim).

Myelocyten mit pseudoeosinophilen Granulationen sind selten zu finden, so daß auf ihr Vorhandensein im Verlauf dieser Erörterungen kein großer Wert gelegt werden soll. Megakariocyten wurden nicht beobachtet.

Hier muB auf noch eine Zell- resp. Kernform eingegangen werden, die man ziemlich häufig trifft; sie soll hier nur verzeichnet werden, ohne daß ein abschließiendes Urteil über ihre Bedeutung abgegeben werden 
soll. Im Schnitt erscheint der Kern hochgradig pyknotisch und in eine Anzahl ungleich großer, zum Teil bizzar geformter Fragmente zersprengt. In Abstrichpräparaten erscheinen diese Kernkonfigurationen zum Teil auffallend blaß, z. B. im Vergleich mit pyknotischen Erythroblastenkernen, zeigen oft Kleeblattfiguren, z. T. sind sie ebenfalls intensiv dunkel gefärbt, wie stark verdichtetes Chromatin und sehen wie zerbröckelt aus. $\mathrm{Da}$, wie besonders aus Abstrichpräparaten hervorgeht, diese Kernstrukturen hauptsächlich in hämoglobinhaltigen Zellen vorkommen, dürfte die Frage berechtigt sein, ob es sich bei diesen Zellen nicht um einen auf physiologischem Kernschwund beruhenden Vorgang handelt, also von Übergangsstadien von Erythroblasten zu Erythrocyten. Selbstverständlich könnten diese Bilder auf rein pathologisch degenerativen durch Giftwirkung zu erklärenden Veränderungen beruhen, wodurch ihre Bedentung für die vorliegenden Erörterungen natürlich hinfällig werden würde.

Das Verhalten der Endothelzellen ist in mehrfacher Hinsicht von Interesse. An vielen Stellen scheinen sie wie abgelöst von den Leberbalken, and zwar augenscheinlich besonders dort, wo sich die oben beschriebenen Zellhaufen in den lakunär ausgeweiteten Capillarsinussen finden ${ }^{1}$. In diesen Partien sieht man bei sehr dünnen Schnitten und tadelloser Konservierung und Färbung von einem derart abgehobenen Endothelstreifen einzelne feine Fasern ausgehen, die stellenweise kaum sichtbare Maschen mit einander bilden. Zum Teil in diesen Maschen, zum Teil mit ihnen in engster Berübrung liegen nun die verschiedenen oben beschriebenen Zellen. Stellenweise scheint eine feinste aus einem solchen Netzwerk hervorgehende Faser wie mit einem Fortsatz an einer Zelle zu haften.

Einzelne Endothelien enthalten eosinophile resp. pseudoeosinophile Granula, was wohl anf ihre phagocytäre Tätigkeit zu beziehen ist.

Mit Rücksicht auf die von M. B. Schmidt ${ }^{2}$ ) erhobenen Befunde zahlreicher Mitosen an dən Endothelien embryonaler Lebern und Milzen, muß hier bemerkt werden, daß ein derartiger $z$ wingender Beweis für autochtone Zellproliferation in den vorliegenden Fällen nicht erhoben werden konnte.

Endlich ist noch als beiläufiger Befund zu erwähnen, daß, übereinstimmend mit den von M. B. Schmidt an Embryonen erhobenen Befunden, die in den Capillaren vorhandenen roten Blutkörperchen auffallend verschieden große Durchmesser haben, ohne daß eine Congruenz mit den Befunden im zirkulierenden Blut zu bestehen braucht.

Um bei der oben geschilderten Schwierigkeit bezügl. der Lokalisation der in Frage kommenden Zellen ïber ibre intra- resp. extracapilläre Lage sicher urteilen zu können, wurde versucht, mit Hilfe der Methode von Maresch ${ }^{3}$ ) eine Silberimprägnation der feinsten oben

1) Da die allermeisten Tiere nach dem Tode im Laufe der nächsten Stunde zur Sektion kamen, dürften kadaveröse Gewebsveränderungen auszuschließen sein.

2) Zieglers Beiträge, Bd. 11, 1892.

3) Zentralbl. f. allgem. Pathol., Bd. XVI, Nr. 16 und 17. 
beschriebenen Fasern vorzunehmen, jedoch konnte auf diesem Wege kein positives Ergebnis erzielt werden.

Das numerische Verhältnis der verschiedenen Zellarten im Leberabstrich ergab im Falle $\mathrm{Nr}, 20$ :

$\begin{array}{lr}\text { Erythroblasten } & 14,8 \text { Proz. } \\ \text { mononucleäre lymphoide Zellen } & 75,4= \\ \text { pseudoeos. polyn. L. } & 6,2= \\ \text { pseudoeos. Myelocyten } & 3,8= \\ \text { e 21: } & 12,9= \\ \text { Erythroblasten } & 79,8= \\ \text { mononucl. lymph. Z. } & 6,3= \\ \text { pseudoeos. polyn. L. } & 2,0= \\ \text { pseudoeos. Myelocyten } & \end{array}$

Die mikroskopische Untersuchung der Milz ergab eine ziemlich starke Verkleinerung der Follikel, die wohl als Druckatrophie aufgefaßt werden mu, da gleichzeitig eine Wucherung der Pulpa vorlag.

Eine genauere Analyse der Pulpazellen zeigt, daß es sich nicht um eine eigentliche myeloide Umwandlung handelt, wie sie bei der myeloiden Leukaemie die Regel ist, wenn man die leukoblastische Funktion an der Zahl der Myelocyten bemißt. Dagegen finden sich unverkennbar herdförmige Erythroblastenhaufen vor. Diese scheinen keinen eigentlichen Prädilektionsort in Bezug auf Trabekel usw. zu haben, wie das z. B. Eliasberg in seinen Versuchen beobachtete. Dagegen ist erwähnenswert, daß die meisten Bill rothschen Venensinus mit kernhaltigen roten Blutkörperchen wie vollgestopft erscheinen, wobei natïrlich auch die großen einkernigen Zellen mit Buchtkern und breitem ungranuliertem Protoplasma ("Splenocyten") nicht fehlen. Mitosen an den Endothelien wurden nicht beobachtet. (Fig. 4).

Auch hier drängt sich einem besonders bei Übersichtspräparaten der Antagonismus zwischen den zellulären Pulpaelementen resp. ihren Derivaten, und den lymphoiden Follikein auf. Letztere erscheinen bei der unter dem Einfluß der Anämie einsetzenden Metaplasie des Milzgewebes wie unbeteiligt und der Expansion der wuebernden Pulpa hinderlich.

Selbstverständlich finden sich massenhaft pigmenthaltige Makrophagen vor, die verschiedene Phasen im Abbau der roten Blutkörperchen vom fast intakten Erythrocyten herab bis zur gelben Pigmentscholle in sich vereinigen.

Da somit die histologischen Details der Milz im großen und ganzen Bekanntes darbieten, sei hier auf weitere eingehende Beschreibung verzichtet. Im Abstrichpräparate finden sich selbstverständlich in der großen Mehrzahl Zellen von typisch-lymphoidem Habitus, große Lymphocyten, kleine Lymphocyten und wieder breitrandige granulafreie Zellen mit eingebuchtetem Kern. Daneben ein erbeblicher Prozentsatz kernbaltiger Erythrocyten teils mit pyknotischen, teils mit nichtpyknotischen Kernen, auch hier nur spärlich Myelocyten.

Auch das Knochenmark zeigt typischen Befund wie bei jeder schwereren Anaemie (Zellmark nach Schur-Löwy), so daß auch hier 
eine kurze Beschreibung genügt. Es handelt sich im wesentlichen um vorzugsweise Proliferation der Erythrocyten und Lymphoid. zellen, während die Ganulocyten an Zahl zurücktreten. Die im normalen Mark charakteristischen Fettgewebslücken sind völlig geschwunden. Zahlreiche pigmenthaltige Phagocyten finden sich in jedem Gesichtsfeld; ebenso ist die Zahl der Knochenmarksriesenzellen nicht unbedeutend.

Den beiden erwähnten Fällen sei ein dritter ähnlicher angereiht, der wegen seines völlig analogen Verhaltens nur ganz kurz berührt werden soll.

Männl. Kaninchen Nr. 10. Gewicht $1550 \mathrm{~g}$.

Blutbefund am 19. V. 1906: 90 Proz. Hb; 7630000 R.; 9900 L.; erhält in der Zeit vom 19. V. bis 10. VI. im ganzen 0,34 Phenylhydrazinacetat subkutan und hat am 10. VI. folgenden Blutbefund: 20. Proz. Hb; 1650000 R.; 9600 L. (keine kernhaltigen Roten, an den Tagen zuvor spärliche Erythroblasten.)

Auch hier Leber und Milz, wenn auch in geringerem Grade, wie in den vorigen Fällen verändert. In beiden Organen zahlreiche Erythroblasten, vereinzelte Myelocyten.

Endlich soll ein 4. Fall wiedergegeben werden, der seines eigentiimlichen Milzbefundes wegen von Interesse ist.

Männl. Kaninchen Nr. 8; Gewicht 1850; Pyrogallollösung 1) 10 proz. subkutan.

Blutbefund:

28. IV. 06. 5 Uhr. 110 Proz. Hb (Sahli) 6930000 R.; 13400 L. 0,1.

29. IV. 11 Uhr a. m. 95 Proz. Hb; 5930000 R.; 12900 L. 0,1.

30. IV. 11 Uhr a. m. 85 Proz. Hb; 5000000 R.; 11400 L.

2. V. 10 Uhr a. m. 80 Proz. Hb; 5180000 R.; 10300 L. 0,1 .

5. V. 7 Uhr p. m. 80 Proz. Hb; 4500000 R.; 8500 L. $0,1$.

6. V. 11 Uhr a. m. 75 Proz. Hb; 3990000 R.; 8700 L. 0,05.

7. V. 7 Uhr p. m. 80 Proz. Hb; 4870000 R.; 8900 L. 0,1 .

9. V. 12 Uhr a. m. 90 Proz. Hb; 5500000 R.; 10000 L. 0,2 .

10. V. 12 Uhr m. - $\quad 5200000$ R.; 14600 L. 0,2 .

11. V. 12 . Uhr m. - - - 0,1 .

5 Uhr p. m. 75 Proz. Hb; 4870000 R.; 5900 L. 0,1.

12. V. 9 Uhr a. m. 75 Proz. Hb; 5000000 R.; 17800 L. -

14. V. 9 Uhr a. m. 75 Proz. Hb; 5200000 R.; 14400 L. 0,2.

15. V. 9 Uhr a. m. 60 Proz. Hb; 4100000 R.; 11200 L. 0,4.

16. V. 9 Uhr a. m. 55 Proz. Hb; 3800000 R.; 21900 L.

12 Uhr a. m. - - - $\quad 0,4$.

17. V. 9 Uhr a. m. 55 Proz. Hb; 3670000 R.; 17700 L. 0,5 .

18. V. 11 Uhr a. m. 58 Proz. Hb; 3800000 R.; 7800 L. 0,4.

19. V. 11 Uhr a.m. 60 Proz. Hb; 3120000 R.; 14100 L. 0,3.

20. V. 11 Uhr a. m. 52 Proz. Hb; 2350000 R.; 14900 L. 0,4.

1) Da ein und dieselbe Pyrogallollösung ziemlich lange in Gebrauch war, ist anzunehmen, daB der toxische Wert jeder einzelnen Dosis im Laufe der Zeit geringer wurde, als den im Protokoll notierten Dosen entspricht.

Archiv f. experiment. Pathol. u. Pharmakol. Bd. 58. 
21. V. 10 Uhr a. m. 50 Proz. Hb; 2200000 R.; 19200 L. 0,5.

22. $V$.

23. V. 11 Uhr a. m. 50 Proz. Hb; 2300000 R.; 17400 L. $0,3$.

24. V. 12 Uhr m. 48 Proz. Hb, 2100000 R.; 8200 L. $0,4$.

25. V. 6 Uhr p. m. 50 Proz. Hb; 2400000 R.; 8400 L. $0,5$.

26. V. 11 Uhr a. m. 40 Proz. Hb; 1990000 R.; 6500 L. $0,5$.

27. V. 12 Uhr m. 35 Proz. Hb; 1780000 R.; 3200 L. $0,6$.

28. V. 12 Uhr m. 30 Proz. Hb; 1200000 R.; 9800 L. 0,5 .

29. V. 8 Uhr p. m. 25 Proz. Hb; 1420000 R.; 9100 L. 0,5.

30. V. 11 Uhr a. m. 23 Proz. Hb; 1310000 R.; 4900 L. 0,3.

30. V. 12 Uhr m. wird das Tier durch Schlag auf den Kopf getötet.

Die Sektion ergibt im wesentlichen einen sehr ähnlichen Befund wie die beiden vorigen Fälle, die Leber ist womöglich noch stärker verändert, wenigstens finden sich noch reichlichere intravasculäre Zellaggregate, wenn auch die Neigung circumscripte Herde zu bilden weniger ausgebildet ist.

Das Leberabstrich präparat enthält 16 Proz. Erythroblasten. Die übrigen histologischen Details weichen fast nicht von den vorhergehenden Fällen ab.

Anders steht es in diesem Falle mit der Milz. Sie ist ebenfalls wieder stark vergrößert, ihre Maße sind $7 \times 1 \times 0,5 \mathrm{~cm}$; die Pulpa dunkel rotbraun, schmierig weich. Der Schnitt weist ein Bild auf, wie es nur in diesem einzigen Falle angetroffen wurde, während in allen übrigen Fällen auch nicht andeutungsweise eine ähnliche Veränderung beobachtet wurde.

Das Auffallendste bei schwacher Vergrößerung ist ein derartig hochgradiger Schwund alles lymphoiden Gewebes inklusive Follikel, daß nur ganz spärliche Reste von diesem übrig sind, ja stellenweise um ein Follicular-Gefäß herum nur noch kleinste Zellhäufchen den ehemaligen Follikel andeuten. In entsprechendem Maße sind die lymphoiden Advenditialscheiden der Gefäße auf äußerst geringe Reste reduziert, so daß oft nur wenige in einem Längszug angeordnete Lymphoidzellen übrig sind, die die ehemalige Struktur skizzieren.

Die rote $\mathrm{Pulpa}$ resp. in diesem Fall diejenigen Zellelemente, die sich außer den Follikelresten überhaupt noch im Milzgewebe finden, ist ebenso eingreifend verändert. Bei flichtiger Betrachtung erscheint nämlich dieser Teil eines Milzschnittes wie fast homogen und strukturlos, resp. wie fein gekörnt, ohne jeden sichtbaren Zellgehalt, bezw. frei von wahrnehmbaren Kernen. Bei starker Vergrößerung sucht man vergeblich auch hier nach kernhaltigen Gebilden, tatsächlich handelt es sich um eine geradezu ungeheure Ansammlung von kernlosen roten Blutkörperchen, die infarktartig die rote Pulpa durchsetzen und augenscheinlich alle übrigen zellulären Elemente zum Schwunde gebracht haben.

Das Bindegewebsgerüst, die Trabekel, und die Gefäßwände deuten nicht auf indurative Prozesse. An einzelnen Follikulargefäßen ist hingegen bemerkenswert, daß die Gefäßwände partiell nekrotisch erscheinen und an diesen Stellen eine Blutung in die Follikel zustande gekommen ist: Beachtenswert ist ferner das völlige Fehlen von 
freiem und intrazellulärem Pigment, wie es in allen übrigen mit langdauerndem Blutzerfall einhergehenden Anämien der Fall war. Der Vergleich mit einer Infarktbildung liegt histologisch nahe, doch ist nicht einleuchtend, warum die ganze. Milz derartig gleichmäßig verändert war. Pathologisch-anatomisch war jedenfalls kein Grund für einen derartigen Befund anzugeben.

Vom Knochenmark ist hier nur als beiläufiger Befund zu erwähnen, daß es entschieden mehr Pigment enthält als in den übrigen untersuchten Fällen. Im übrigen ist es typisches vorwiegend erythropoetisches Zellmark.

Weiter seien hier einige Fälle mitgeteilt, bei denen der Verlauf der Anämie etwas modifiziert wurde, durch Einschaltung von Erholungsperioden zwischen die Perioden progredienter Intoxication.

Weibl. Kaninch. Nr. 11 Gewicht $1400 \mathrm{~g} ;$ Phenylhydracinacetat subkutan.

18. VI. 0612 Uhr m. 105 Proz. Hb (Sahli); 7460000 R.;5200 L. 0,01 19. VI. 1 Uhr p. m. 95 Proz. Hb; 6400000 R.; 12400 L. 0,02

20. VI. - - - - 0,02

21. VI. 10 Uhr a. m. 45 Proz. Hb; 4190000 R.; 13900 L. -

22. VI. 11 Uhr a. m. 40 Proz. Hb; 3970000 R.; 10100 L. 0,015

23. VI. $11 \mathrm{Uhr}$ a. m. 35 Proz. Hb; 2590000 R.; 11300 L. 0,015

24. VI. 11 Uhr a. m. 27 Proz. Hb; 1660000 R.; 20460 L. -

25. VI. 10 Uhr a. m. 35 Proz. Hb; 2228000 R.; 18600 L. -

26. VI. $11 \mathrm{Uhr}$ a. m. 45 Proz. Hb; $2820000 \mathrm{R}$; $12700 \mathrm{~L}$. -

27. VI. $12 \mathrm{Uhr}$ m. 50 Proz. Hb; 3500000 R.; $8800 \mathrm{~L}$. -

28. VI. 11 Uhr a. m. 60 Proz. Hb; 3320000 R.; 8900 L. -

29. VI.

30. VI. 11 Uhr a. m. 80 Proz. Hb; 4690000 R.; 4500 L. -

1. VII. 10 Uhr a. m. - - - 0,02

2. VII. 11 Uhr a. m. 68 Proz. Hb; 4470000 R.; 6000 L. -

4. VII. $11 \mathrm{Uhr}$ a. m. - - 0,02

5. VII. 11 Uhr a. m. 55 Proz. Hb; 3440000 R.; 5300 L. 0,01

6. VII. 11 Uhr a. m. 52 Proz. Hb; 3200000 R.; 6000 L. 0,015

7. VII. 11 Uhr a. m. 48 Proz. Hb; 3110000 R.; 15000 L. 0,02

8. VII.

9. VII. 11 Uhr a. m. 43 Proz. Hb; 2360000 R.; 17000 L. 0,005

10. VII. 12 Uhr m. 40 Proz. Hb; 2110000 R.; 10200 L. 0,01

11. VII. 11 Uhr a. m. 35 Proz. Hb; 2210000 R.; 9400 L. 0,01

12. VII. 11 Uhr a. m. 27 Proz. Hb; 1950000 R.; 7900 L. -

13.-16. VII.

16. VII. 11 Uhr a. m. 60 Proz. Hb; 4950000 R.; 6800 L. -

12 Uhr m. getötet.

Das Prozentualverhältnis der kernhaltigen Zellen im Blut: Lymphoide Formen 74,4 Proz.,

Pseudoeos. polyn. L. 18,8 Proz.,

Eosinoph. polyn. L. 2,7 Proz.,

Mastzellen

Erythrobl.

Myelocyten

4 Proz.,

0 Proz.,

0 Proz. 
Ein Paralleltier vom selben Wurf wie Nr. 9, wurde derselben Versuchsanordnung unterworfen.

Weibl. Kaninchen Nr. 12. Gew. 1250 g. Phenylhydracinacetat subkutan.

Blutbefund:

19. VI. 06. 90 Proz. Hb; 6250000 R.; 9700 L. 0,01

20. VI.

21. VI. 11 Uhr a. m. 50 Proz. Hb; 4820000 R.; 8400 L. 0,02

22. VI. 11 Uhr a. m. 50 Proz. Hb; 4220000 R.; 14900 L. 0,02

23. VI. 11 Uhr a. m. 42 Proz. $\mathrm{Hb} ; 4000000$ R.; 12800 L. 0,01

24. VI. 10 Uhr a. m. 38 Proz. Hb; 3750000 R.; 21200 L. 0,015

25. VI. 11 Uhr a. m. 28 Proz. Hb; 3820000 R.; 17400 L. 0,015

26. VI. 11 Uhr a. m. 22 Proz. Hb; 2790000 R.; 4800 L. -

27. VI. 10 Uhr a. m. 28 Proz. Hb; 1740000 R.; $10400 \mathrm{~L}$. -

28. VI. 11 Uhr a. m. 30 Proz. Hb; 1630000 R.; 5700 L. -

29. VI. 10 Uhr a."m. 36 Proz. Hb; 2500000 R.; 5300 L. -

30. VI. 11 Uhr a. m. 40 Proz. Hb; 2520000 R.; 7700 L. -

1. VII.

2. VII. 10 Uhr a. m. 66 Proz. $\mathrm{Hb} ; 4470000$ R.; 5900 L. -

3. VII.

4. VII. 11 Uhr a. m. 80 Proz. Hb; 5600000 R.; 6600 L. 0,015

5. VII. 11 Uhr a. m. 55 Proz. Hb; 4460000 R.; 8500 L. 0,01

6. VII. 10 Uhr a. $\mathrm{m}, 68$ Proz. $\mathrm{Hb} ; 5230000$ R.; 7000 L. 0,015

7. VII. 11 Uhr a. m. 52 Proz. Hb; 4400000 R.; 10000 L. 0,02

9. VII. 11 Uhr a. m. 45 Proz. Hb; 3510000 R.; 9900 L. 0,01

11. VII. 10 Uhr a. m. 50 Proz. $\mathrm{Hb} ; 2910000$ R.; 6500 L. 0,02

12. VII. 11 Uqr a. m. 35 Proz. Hb; 1870000 R; 9900 L. 0,01

13. VII. 11 Uhr a. m. 22 Proz. Hb; 1900000 R.; 8700 L. -

14. VII. $10 \mathrm{Jhr}$ a. m. 20 Proz. Hb; 1670000 R.; $7200 \mathrm{~L}$. -

16. VII. $10 \mathrm{Uhr}$ a. m. 30 Proz. $\mathrm{Hb} ; 2510000 \mathrm{R}$; $7200 \mathrm{~L} . \quad$

17. VII. 11 Uhr a. m. $50 \mathrm{Prz} . \mathrm{Hb}($ ?); 3900000 R.; 6400 L. -

17. VII. 2 Uhr p. m. wird das Tier getötet.

Auszählung des Blutabstrichpräparates ergibt:

$\begin{array}{lr}\text { Lymphoide Formen } & 64,2 \text { Proz. } \\ \text { Pseudoeos. polyn. L. } & 23,6= \\ \text { Mastzellen } & 7,8= \\ \text { Eos. polyn. L. } & 4,1= \\ \text { Erythrobl. } & 0,5= \\ \text { Pseudoeos. Myelocyten } & -=\end{array}$

Das Sektionsergebnis beider Tiere soll hier soweit wiedergegeben werden, als es von dem der vorher genannten Fälle abweicht.

Für die Leber ist hervorzuheben, daß die oben beschriebenen Zellen in exquisiter Weise Neigung zeigen, Gruppen zu bilden (siehe Fig. 1), deren einzelne Zellen so dicht beieinander liegen, daß man über ihr Verhältnis $z u$ den Lebercapillaren nichts Sicheres aussagen kann, und auch bei starker Vergrößerung ist es unmöglich zu entscheiden, was innerhalb und was außerhalb der Capillarwand liegt. Auch hier soll besonders auf die Tatsache der Herdbildung Nachdruck 
gelegt werden. Sicher zeigen rein quantitativ die beiden letzten Fälle hierin stärkere Veränderungen. Dem entspricht auch ein höherer Gehalt an Erythroblasten im Ausstrichpräparat der Leber (s. Fig. 2).

Bei Nr. 11: 25,0 Proz. Erythroblasten,

$$
\begin{aligned}
2,4 & =\text { Myelocyten, } \\
\text { 12: } & =\text { Erythroblasten, } \\
1,9 & =\text { Myelocyten. }
\end{aligned}
$$

Auch die Milz verhält sich von den vorigen Fällen abweichend. Schon bei schwacher Vergrößerung sieht man zablreiche Zellen in der Pulpa, teils einzeln, teils gruppenförmig zusammen liegend, die deutlich eosinophile Granulationen besitzen, bei starker Vergrößerung erkennt man sie als hauptsächlich eosinophile Myelocyten. Ihrer Lage nach scheinen sie stellweise die perivasculären Pulpaschichten zu bevorzugen. An anderen Stellen läßt sich keine Regel für ihre Lokalisation auffinden.

Nie wurden - auch bei sorgfältigstem Suchen - Myelocyten im Bereich der Malpighischen Körperchen gefunden.

Daneben sind wieder zahlreiche kernhaltige rote Blutkörperchen in Gruppen und vereinzelt in der Pulpa zu finden, die Venensinus sind z. T. mit mononucleären, teilweise haemoglobinhaltigen Zellen erfüllt, doch sind diese Bilder lange nicht so häufig wie bei den erstgenannten Fällen.

Hier soll übrigens bemerkt werden, daß das Knochenmark im Gegensatz zu den Fällen 20, 21 und 10 zweifellos reicher an granulierten Zellen ist und daß speziell die pseudoeosinophilen Myelocyten in den beiden letzten Fällen nicht unwesentlich stärker vertreten sind. ${ }^{1}$ )

Greift man die wesentlichen Punkte der vorliegenden Untersuchungeu heraus, so ergibt sich, daß sich bei künstlieher Anämisierung von Kaninchen vermittelst hämolytisch wirkender Gifte in Milz und Leber Veränderungen erzielen lassen, wie sie unter normalen Verhältnissen beim ausgewachsenen Tiere niemals anzutreffen sind, die aber eine nicht zu verkennende $\ddot{A}$ hnlichkeit mit Bildern aufweisen, die Erich Meyer und A. Heineke bei schweren menschlichen Anämien in den genannten Organen beschrieben haben (Fig. 4).

Dabei ist augenscheinlich für ihr Zustandekommen im Tierexperiment chroniseher Verlauf der Anämie eine notwendige Bedingung, wie das aus dem negativen Resultat der drei erst angeführten Versuche (Tiere Nr. 2, 3, 15) hervorgeht; zweitens weisen unter der Zahl der ehro-

1) Dieser Befund im Hinblick auf die in diesem Falle konstatierte ausgeprägte myeloide Metaplasie des Markes ist eine Illustration für die von Pappenheim früher speziell für die Leukaemie gemachte Beobachtung, daß Knochenmarl und myeloid metaplasierte Milz cytologisch einander korrespondieren. 
nischen lang ansgedehnten Anämien wiederum diejenigen die stärkeren Veränderungen auf, bei denen periodenweise eiqn Absehnitt von kontinuierlicher Giftzufuhr und entsprechend progressiver Anämie mit einem Erholungsstadium abwechselte.

Dieses Verbalten spricht eindeutig in dem Sinne, daß die hier experimentell hervorgerufenen Organveränderungen als Zeichen der Regeneration aufzufassen sind. Es fragt sich nur, ab alle in Leber und Milz vorhandenenen jugendlichen Blutzellen an Ort und Stelle gebildet worden sind, oder ob sie aus anderen Organen, dem Knochenmark, eingeschwemmt, in Leber und Milz nur zu reifen Elementen sich entwickeln. Daß in diesen Organen eine lebhafte Zellbildung besteht, zeigen die relativ zahlreichen Mitosen an den indifferenten Blutkörperchen und den Erythroblasten. Im zirkulierenden Blut wurden niemals Mitosen gesehen. Ferner spricht die ausgesprochene herdweise Gruppierung der jungen Zellen sowohl in der Leber wie in der Milz mehr für eine lokale Bildung, als für Einschwemmung.

Eine besonders wichtige Tatsache ist der wesentlieh verschiedene Gehalt an kernhaltigen Erythrocyten in den Abstrichen der Organe, speziell der Leber, einerseits und den kurz vor dem Tode entnommenen Blutabstrichpräparaten andrerseits. $\left.{ }^{1}\right)$ Die Resultate seien hier im Auszug wiederholt:

Tier Nr. 20, Blutpräparat vom 15. V.: Erythrobl. im Leberabstrichpräparat Erythrobl.

Tier Nr. 21, Blutpräparat (13. V.): 2,2 Proz.

$14,8=($ Fig. 3)

Leberabstrich

Erythrobl.

Tier Nr. 11, Blut (16. VII).:

Leberabstrich

Tier Nr. 12, Blut (17. VII.):

Leberabstrich

$\begin{aligned} 0,5 & = \\ 12,9 & = \\ - & = \\ 25,0 & = \\ 0,5 & = \\ 38,2 & =\end{aligned}$

Endlich muß, anch in den am Tier gewonnenen Befunden auf die frappante Ähnlichkelt mit den Bildern in den gleichen fötalea

1) Es soll ausdrücklich betont werden, was übrigens aus den Protokollen hervorgeht, daß man sich durch tägliche Kontrolle des Blutbildes darüber vergewisserte, daß ein Tier nicht gerade in der Periode zur Untersuchnng gelangte, wo im Blut eben ein Schwarm Erythroblasten nachweisbar war und durch Einschwemmung in die verschiedenen Organe zu Tăuschungen Veranlassung geben konnte. Dieselbe Vorsicht wurde übrigens auch gegenüber hohen Lenkocytenwerten beobachtet, was einer der Gründe für die erhebliche Reduktion der Zahl verwertbarer Versuche ist. 
Organen aufmerksam gemacht werden, an die Meyer und Heineke hauptsächlich ibre theoretische Überlegungen knüpfen: Hier wie dort in den Lebercapillaren zahlreiche Zellen, die teils echte Erythroblasten, teils Lymphoidzellen sind, bei denen hier wie dort zum Teil nicht zu entscheiden ist, was intra- und was extracapillär liegt.

Schließlich aber beansprucht eine Beobachtung - und diese darf durch einen Teil der vorliegenden Untersuchungen als erwiesen gelten - besondere Beachtung, das ist die Tatsache, daß die stärksten Veränderungen in den Organen resp. der höchste Gehalt an Jugendformen der roten Blutkörperchen sich bei denjenigen Tieren fanden (Nr. 11 nnd 12), bei denen im Verlauf der Vergiftung giftfreie Erholungspausen eingeschaltet wurden, bei denen also dem Organismus Gelegenheit gegeben wurde, mit genügender Kraft auf die Schädigang: des Blutes zu reagieren. Daß er es mit Erfolg tat, geht aus dem jedesmaligen rapiden Ansteigen der Werte von Erythrocyten und Hämoglobin hervor.

\section{Zusammenfassung.}

Dureh protahierte Vergiftung mit Phenylhydrazin, Pyrogallol usw. können bei Kaninchen Organveränderungen hervorgerufen werden, die mit denen mensehlicher perniciös anämiseher Organe die weitgehendste Ähnlichkeit besitzen. Diese bestehen in lymphoider Umwandlung des Knochenmarkes, myeloider Umwandlung der Milz mit Erythro- und Leukopoese, und in dem Auftreten von Knochenmarkselementen in der Leber. Diese Veränderungen kommen nur dann zustande, wenn die Vergiftung eine chronische ist; sie sind am stärksten ausgebildet, wenn das Tier sich wäbrend der Vergiftung mehrmals erholt hatte und der Vergiftung erst allmählich erlag; sie fehlen immer bei akuter Vergiftung. Dies Verhalten spricht für die von Erich Meyer und A. Heineke ausgesprochene Annahme, daß die gleichen bei Menschen mit perniciöser Anämie gefundenen Organveränderungen ein Ausdruck der Regenerationbestrebung des Organismus gegen gesteigerten Blutzerfall bedeuten. Auch beim Tier ähneln die beschriebenen Veränderungen aufs Deutlichste den Organbefunden embryonaler Tiere. Auch hierin liegt eine weitere Analogie zu den Organbefunden der perniciösen Anämie beim Menschen.

Nachtrag zur Korrektur.

Aus äußeren Gründen konnte die vorliegende Arbeit, die bereits Juli 1907 fertiggestellt war, nicht früher veröffentlicht werden. Unterdessen ist die Arbeit von Blumenthal und Morawitz (Ex- 